\title{
A GENERALIZATION OF A PROPERTY OF HARMONIC FUNCTIONS*
}

\author{
MAX CORAL
}

1. Introduction. A well known theorem of Bôcher and Koebe characterizes a function $u(x, y)$ as harmonic in a region $A$ if $u$ is of class $C^{\prime}$ in $A \dagger$ and if the integral of its normal derivative is zero around every circle $C$ in $A$. This theorem has been generalized by Gergen $\ddagger$ as follows:

If $v(x, y)$ is harmonic and positive in $A$, if $u(x, y)$ is of class $C^{\prime}$ in $A$, and if

$$
\int_{C} v \frac{\partial u}{\partial n} d s=\int_{C} u \frac{\partial v}{\partial n} d s
$$

for every circle $C$ interior to $A$, then $u$ is harmonic in $A$.

The Bôcher-Koebe result is secured from Gergen's theorem by choosing $v(x, y) \equiv 1$ in $A$. Gergen's theorem in turn is a special case of the following theorem concerning a general linear partial differential equation of the second order which is self-adjoint and of elliptic type:

TheOREM 1. Consider the differential expression

$$
L(z)=a z_{x x}+2 b z_{x y}+c z_{y y}+d z_{x}+e z_{y}+f z
$$

whose coefficients $a, b, \cdots, f$ are functions of $(x, y)$ of class $C^{\prime \prime}$ in $A$ which satisfy the conditions

$$
a_{x}+b_{y}=d, \quad b_{x}+c_{y}=e, \quad a c-b^{2}>0
$$

in A. Let

$$
\lambda(z)=a z_{x}+b z_{y}+d z, \quad \mu(z)=b z_{x}+c z_{y}+e z .
$$

Let $v(x, y)$ be a function of class $C^{\prime \prime}$ which never vanishes in $A$ and which satisfies $L(v)=0$. If $u(x, y)$ is of class $C^{\prime}$ in $A$, and if

* Presented to the Society, October 28, 1933, under the title, $A$ property of selfadjoint elliptic partial differential equations.

$\dagger$ A function $u(x, y)$ is of class $C^{(n)}$ in $A$ if it is continuous and has continuous partial derivatives in $A$ of all orders up to and including the $n$ th.

$\ddagger \mathrm{J}$. J. Gergen, Note on a theorem of Bôcher and Koebe, this Bulletin, vol. 37 (1931), pp. 591-596. See also S. Saks, Note on defining properties of harmonic functions, this Bulletin, vol. 38 (1932), pp. 380-382. 


$$
\int_{C} v[\lambda(u) d y-\mu(u) d x]=\int_{C} u[\lambda(v) d y-\mu(v) d x]
$$

for every circle $C$ in $A$, then $u(x, y)$ is of class $C^{\prime \prime}$ in $A$ and $L(u)=0$.

2. The Haar fundamental lemma. Theorem 1 is capable of rather simple proof if use is made of the so-called fundamental lemma of Haar* for the calculus of variations of a double integral. We shall establish Haar's lemma in a form adapted to our purposes:

Theorem 2. Let $P(x, y), Q(x, y)$, and $T(x, y)$ be continuous in a bounded region $A$. If the integral

$$
\iint_{A}\left(P \zeta_{x}+Q \zeta_{y}+T \zeta\right) d x d y
$$

has the value zero for every function $\zeta(x, y)$ of class $C^{\prime}$ in $A$ which vanishes on the boundary of $A$, then

$$
\int_{C} P d y-Q d x=\iint_{C} T d x d y
$$

on every circle $C$ interior to $A$. Conversely, if (7) holds on every circle $C$ interior to $A$, and if $D$ is a region interior to $A$ which is bounded by a simply closed regular curve $\Delta$, then

$$
\iint_{D}\left(P \zeta_{x}+Q \zeta_{y}+T \zeta\right) d x d y=0
$$

for every function $\zeta(x, y)$ of class $C^{\prime}$ in $D$ which vanishes on $\Delta$.

Let $C$ be a circle interior to $A$ with center at $\left(x_{0}, y_{0}\right)$ and radius $r$, and consider the ring $\Gamma$ defined by

$$
\begin{aligned}
& x=x_{0}+\rho \cos \theta, \\
& y=y_{0}+\rho \sin \theta, \quad 0<r_{1} \leqq \rho \leqq r ; 0 \leqq \theta \leqq 2 \pi .
\end{aligned}
$$

If $R(\rho),\left(r_{1} \leqq \rho \leqq r\right)$, is any function of class $C^{\prime}$ for which $R\left(r_{1}\right)=R(r)=0$ and for which the derivative $R^{\prime}(\rho)$ also vanishes for $\rho=r_{1}$ and $\rho=r$, define

$$
\zeta \equiv\left\{\begin{array}{lll}
0 & \text { on } A-\Gamma, \\
R & \text { on } \Gamma .
\end{array}\right.
$$

* A. Haar, Über eine Verallgemeinerung des Du Bois-Reymondsche Lemmas, Acta Litterarum ac Scientiarum, vol. 1 (1922), p. 33. Cf. also A. Haar, Zur Variationsrechnung, Abhandlungen aus dem Mathematischen Seminar der Hamburgischen Universität, vol. 8 (1930), pp. 1-27. 
The function $\zeta(x, y)$ so defined is of class $C^{\prime}$ on $A$ and vanishes on the boundary of $A$; and

$$
\begin{aligned}
& \iint_{A}\left(P \zeta_{x}+Q \zeta_{y}+T \zeta\right) d x d y \\
&=\int_{0}^{2 \pi} \int_{r_{1}}^{r}\left(P R^{\prime} \cos \theta+Q R^{\prime} \sin \theta+T R\right) \rho d \rho d \theta
\end{aligned}
$$

the arguments in $P, Q$, and $T$ on the right-hand side of this equality being the values (8) belonging to $\Gamma$. The hypothesis that the integral (6) vanishes for every function $\zeta(x, y)$ of class $C^{\prime}$ which vanishes on the boundary of $A$ thus implies that

$$
\int_{r_{1}}^{r}\left\{R^{\prime} \int_{0}^{2 \pi}(P \cos \theta+Q \sin \theta) d \theta+R \int_{0}^{2 \pi} T d \theta\right\} \rho d \rho=0
$$

for every function $R(\rho)$ of the type described above. By Du BoisReymond's lemma* it follows that

$$
\left.\int_{0}^{2 \pi}(P \cos \theta+Q \sin \theta) \rho\right|_{\rho=r_{1}} ^{\rho=r} d \theta=\int_{0}^{2 \pi} \int_{r_{1}}^{r} T \rho d \rho d \theta .
$$

Letting $r_{1}$ approach zero, one secures the equality (7).

Suppose, conversely, that (7) holds on every circle $C$ interior to $A$. Let the minimum distance of $\Delta$ from the boundary of $A$ be $2 r>0$. If $C_{x y, \rho}$ represents a circle whose center is at a point $(x, y)$ of the closed region $D+\Delta$ and whose radius is $\rho<r$, then the functions

$$
\begin{aligned}
\pi_{\rho}(x, y) & \equiv \iint_{C_{x y, \rho}} P(x+\xi, y+\eta) d \xi d \eta, \\
\kappa_{\rho}(x, y) & \equiv \iint_{C_{x y, \rho}} Q(x+\xi, y+\eta) d \xi d \eta, \quad(x, y) \text { in } D+\Delta, \\
\tau_{\rho}(x, y) & \equiv \iint_{C_{x y, \rho}} T(x+\xi, y+\eta) d \xi d \eta,
\end{aligned}
$$

are of class $C^{\prime}$ in $D+\Delta$ and have derivatives which are given by

$$
\pi_{\rho x}=\int_{C_{x y, \rho}} P d \eta, \quad \pi_{\rho y}=-\int_{C_{x y, \rho}} P d \xi,
$$

* Cf. A. Huke, An historical and critical study of the fundamental lemma of the calculus of variations, in Contributions to the Calculus of Variations, 1930, University of Chicago Press, 1931, p. 86. 
with similar expressions for the derivatives of $\kappa_{\rho}$ and $\tau_{\rho} . \dagger$ Furthermore, an application of the mean value theorem shows that

$$
\begin{aligned}
& \lim _{\rho \rightarrow 0} \frac{1}{\pi \rho^{2}} \pi_{\rho}(x, y)=P(x, y), \\
& \lim _{\rho \rightarrow 0} \frac{1}{\pi \rho^{2}} \kappa_{r}(x y)=Q(x, y), \\
& \lim _{\rho \rightarrow 0} \frac{1}{\pi \rho^{2}} \tau_{\rho}(x, y)=T(x, y),
\end{aligned}
$$

uniformly for $(x, y)$ in $D+\Delta$. Now if $\zeta(x, y)$ is any function of class $C^{\prime}$ in $D$ which vanishes on $\Delta$, then

$$
\begin{aligned}
\iint_{D}\left(P \zeta_{x}+Q \zeta_{y}+\right. & T \zeta) d x d y \\
= & \lim _{\rho \rightarrow 0} \frac{1}{\pi \rho^{2}} \iint_{D}\left(\pi_{\rho} \zeta_{x}+\kappa_{\rho} \zeta_{y}+\tau_{\rho} \zeta\right) d x d y \\
= & \lim _{\rho \rightarrow 0} \frac{1}{\pi \rho^{2}} \iint_{D}\left[\left(\pi_{\rho} \zeta\right)_{x}+\left(\kappa_{\rho} \zeta\right)_{y}\right] d x d y \\
& +\lim _{\rho \rightarrow 0} \frac{1}{\pi \rho^{2}} \iint_{D}\left(\tau_{\rho}-\pi_{\rho x}-\kappa_{\rho y}\right) \zeta d x d y,
\end{aligned}
$$

as can be seen by the use of (12) and an integration by parts. The first term on the right of the last equality in (13) is zero by Green's theorem since $\zeta$ vanishes on $\Delta$; and the second term also vanishes since

$\pi_{\rho x}(x, y)+\kappa_{\rho y}(x, y)-\tau_{\rho}(x, y)=\int_{C_{x y, \rho}}(P d \xi-Q d \eta)-\iint_{C_{x y, \rho}} T d \xi d \eta$

identically for $(x, y)$ in $D+\Delta$ and $0<\rho<r$. Thus the second part of Theorem 2 is established.

3. Proof of Theorem 1. By virtue of the first two equations (3), the equation $L(z)=0$ is the Lagrange equation

$$
\frac{\partial}{\partial x} F_{p}+\frac{\partial}{\partial y} F_{q}-F_{z}=0
$$

$\dagger$ A proof of the analog of this property of the integral means $\pi_{\rho}, \kappa_{\rho}, \tau_{\rho}$ for the case of functions of three variables can be found in O. D. Kellogg, Foundations of Potential Theory, Berlin, 1929, p. 224. 
for the variation problem

$$
\iint_{A} F(x, y, z, p, q) d x d y=\text { minimum }
$$

whose integrand is the quadratic form

$$
F=\frac{1}{2}\left[a p^{2}+2 b p q+c q^{2}+2 d z p+2 e z q+\left(d_{x}+e_{y}-f\right) z^{2}\right],
$$

where we have put $p=z_{x}, q=z_{y}$. Furthermore, if $F_{z}(z), F_{p}(z), F_{q}(z)$ denote the values of these derivatives with the arguments $(x, y, z$, $z_{x}, z_{y}$ ) belonging to $z$, we have

$$
F_{p}(z)=\lambda(z), \quad F_{q}(z)=\mu(z),
$$

and the equation (5) becomes

$$
\begin{aligned}
\int_{C} v\left[F_{p}(u) d y-F_{q}(u) d x\right] & =\int_{C} u\left[F_{p}(v) d y-F_{q}(v) d x\right] \\
& =\iint_{C}\left\{\frac{\partial}{\partial x}\left[u F_{p}(v)\right]+\frac{\partial}{\partial y}\left[u F_{q}(v)\right]\right\} d x d y \\
& =\iint_{C}\left\{u F_{z}(v)+u_{x} F_{p}(v)+u_{y} F_{q}(v)\right\} d x d y \\
& =\iint_{C}\left\{v F_{z}(u)+v_{x} F_{p}(u)+v_{y} F_{q}(u)\right\} d x d y,
\end{aligned}
$$

where we have made use of Green's theorem, equation (14), and a familiar property of the quadratic form $F$, namely,

$$
u F_{z}(v)+u_{x} F_{p}(v)+u_{y} F_{q}(v)=v F_{z}(u)+v_{x} F_{p}(u)+v_{y} F_{q}(u) .
$$

Let $C_{0}$ be any circle interior to $A$. Since (15) holds on every circle $C$ interior to $A$, it follows from Theorem 2 that

$$
\iint_{C_{0}}\left\{F_{p}(u) v \zeta_{x}+F_{q}(u) v \zeta_{y}+\left[F_{z}(u) v+F_{p}(u) v_{x}+F_{q}(u) v_{y}\right] \zeta\right\} d x d y
$$

vanishes for every function $\zeta(x, y)$ of class $C^{\prime}$ in $C_{0}$ and vanishing on the boundary of $C_{0}$. Hence

$$
\iint_{C_{0}}\left[F_{p}(u) \zeta_{x}^{*}+F_{q}(u) \zeta_{y}^{*}+F_{z}(u) \zeta^{*}\right] d x d y
$$

vanishes for every function $\zeta^{*}(x, y)$ of class $C^{\prime}$ in $C_{0}$ which vanishes on the boundary of $C_{0}$, since for any such function $\zeta^{*}$ we may define 
$\zeta \equiv \zeta^{*} / v$ and the integral (17) then reduces to the integral (16). Again using Theorem 2 we find that

$$
\int_{C} F_{p}(u) d y-F_{q}(u) d x=\iint_{C} F_{z}(u) d x d y
$$

for every circle $C$ interior to $C_{0}$. Since $C_{0}$ is any circle in $A$, the result (18) holds for every circle $C$ in $A$.

That the function $u(x, y)$ is of class $C^{\prime \prime}$ in $A$ now follows from a result due to Lichtenstein $\dagger$ since $a c-b^{2}>0$; hence, by Green's theorem,

$$
\begin{aligned}
\iint_{C}\left[\frac{\partial}{\partial x} F_{p}(u)+\frac{\partial}{\partial y}\right. & \left.F_{q}(u)-F_{z}(u)\right] d x d y \\
& =\int_{C}\left[F_{p}(u) d y-F_{q}(u) d x\right]-\iint_{C} F_{z}(u) d x d y=0
\end{aligned}
$$

on every circle $C$ interior to $A$. It follows readily that

$$
L(u)=\frac{\partial}{\partial x} F_{p}(u)+\frac{\partial}{\partial y} F_{q}(u)-F_{z}(u)=0
$$

in $A$, as was to be proved.

WAYNE UNIVERSITY

$\dagger \mathrm{L}$. Lichtenstein, Über den analytischen Charakter der Lösungen regulärer zweidimensionaler Variationsprobleme, Bulletin de l'Académie des Sciences de Cracovie, A, 1912, pp. 915-941. 